\title{
Congenital Varicella Syndrome
}

National Cancer Institute

\section{Source}

National Cancer Institute. Congenital Varicella Syndrome. NCI Thesaurus. Code C116800.

A condition, whose clinical manifestations include intrauterine growth restriction, scarring cicatricial lesion of the limbs, abnormalities of the limbs, microcephaly, chorioretinitis, microphthalmia, cataracts, cortical atrophy, seizures, and evidence of damage to the autonomic nervous system, that is caused by fetal exposure to the varicella zoster virus during the first trimester of pregnancy. 